\title{
Preparation of Hollow Fiber Immobilized Liposome Membrane
}

\author{
Hiroyuki Sugaya ${ }^{1,2)}$, Hiroshi Umakoshi ${ }^{1)}$, Yuji Tohtake ${ }^{1)}$, Ena Oyama ${ }^{1)}$, \\ Toshinori Shimanouchi ${ }^{1)}$, and Ryoichi Kuboi ${ }^{1}{ }^{1}$
}

1) Division of Chemical Engineering, Graduate School of Engineering Science, Osaka University, 1-3 Machikaneyama-cho, Toyonaka, Osaka 560-8531, Japan

2) Specialty Materials Research Laboratories, Toray Industries, Inc., Otsu, Shiga, Japan

Immobilized liposome membrane (ILM) was prepared in hollow fiber module (HF-ILM), where the liposome was loaded into the hollow fiber membrane module and was physically immobilized by forming the hydrogel in the cavity of the fiber membrane. Basic nature of the hydrogels prepared with xanthan gum (XG) and polyethyleneimine (PEI) via amino coupling method was first investigated in relation to the water contents of the gel matrix, so that the optimal condition for the liposome immobilization was obtained. The HF-ILM was prepared at the optimal condition, resulting in the high contents of immobilized liposome with high stability.

Key words : membrane stress biotechnology / hollow fiber membrane / liposome

\section{Introduction}

Liposome, a closed phospholipid bilayer membrane, has a nano-order interface $(\sim 5 \mathrm{~nm})$ harboring hydration layer and low-k (hydrophobic) layer on its surface. It has been reported that the liposome could recognize the molecule through the combined interactions, such as electrostatic and hydrophobic interaction and hydrogen bonds stability ${ }^{1 \sim 3)}$. Some new aspects of the liposome membrane itself, which could be induced under stress condition, have recently been reported ${ }^{4)}$ : (a) molecular chaperone-like function to assist the protein refolding ${ }^{5 \sim 7)}$, (b) protein translocation across the membrane ${ }^{8}$, (c) function as a mediator/initiator of membrane fusion ${ }^{9 \sim 11)}$, (d) LIPOzyme functions (Liposome + Enzyme) ${ }^{12 \sim 19)}$. The use of the liposome (or LIPOzyme) could let us design and develop the liposome-based separation/reactor system.

The immobilization of such a liposome is an important technique for the above-mentioned purposes. It has recently been reported that the liposome can be utilized as a molecular recognition element in several analytical methods, such as (i) immobilized liposome

* Corresponding Author

Tel \& Fax : +81-(0)6-6850-6286

E-mail : MSB@cheng.es.osaka-u.ac.jp chromatography (ILC) / immobilized liposome membrane (ILM) 1, 3, 20) and (ii) immobilized liposome sensor (ILS) ${ }^{21 \sim 23)}$. The above analytical methods enable us to obtain the independent parameters of the liposome molecule interaction, such as charge, hydrophobicity, and hydrogen bond stability. At the same time, the above immobilized liposome techniques can also be applied for the stress-mediated mutual separation of proteins ${ }^{20)}$ and for the protein refolding column ${ }^{1)}$. In these immobilized liposome matrixes, the liposome was immobilized via (a) physical entrapment method ${ }^{24)}$, (b) antigen-antibody method ${ }^{25)}$, (c) hydrophobic ligand method ${ }^{26)}$, (d) covalent binding method ${ }^{27}$ ) and so on. Although these immobilization methods can be utilized for the bioseparation uses, there are some problems such as the capacity of immobilized liposome, the stability of the immobilized liposome, difficulty or non-scalability of the column operation and so on. It is important to develop a new immobilized liposome matrix to overcome the above problems.

Hollow fiber membrane module is widely applied for the bioremediation, water purification, biomedical uses and could be a powerful tool to solve the above problems ${ }^{28)}$. Although a possible use of the planer membrane as a support of the immobilized liposome has been previously reported, the stability of the liposome is not so high to apply it for the practical uses. 
Membrane module, consisted of hollow fiber membranes, has been produced in some membrane companies and utilized in the many research areas ${ }^{29)}$. Considering the morphology of the membrane itself, more effective method for the liposome immobilization could be developed. Polysulfone (PS) hollow fiber membrane, produced in Toray, is a porous membrane and the pore size is varied in a gradual manner (wide from the outside, narrow to the inside of a fiber) ${ }^{30}$. It is expected that the liposome could be easily immobilized through its loading into such a porous space and the following immobilization via polymer gel formation as schematically shown in Fig. 1.

In this study, a method for the liposome immobilization was newly established by using the morphological characteristics of the hollow fiber membrane (HF-M) module produced by Toray. The liposomes were first loaded into the porous space of the membrane module and the hydrophilic gel matrix was formed inside a porous space. The basic characteristics of the hollow fiber type immobilized liposome membrane (HF-ILM) were finally investigated, focusing on the immobilized amounts of liposome, the stability of the immobilized liposome and the characteristics of the membrane module itself.

\section{Experimental}

\subsection{Materials}

1-Palmitoyl-2-oleoyl-sn-glycero-3-phosphocholine (POPC) was purchased from NOF Co. Ltd. (Nagoya, Japan). Polyacrylate (PA), hyalurolic acid (HA), and xanthan gum (XG), used for the hydrophobic $\mathrm{COOH}-$ harboring polymer, were purchased from the WakoPure Inductries (Osaka, Japan). Carboxymethyl-cellulose (CMC, product ID: 039-01335) was also purchased from Wako. Polyethyleneimine (PEI), used for the hydrophobic $\mathrm{NH}_{2}$-harboring polymer, were purchased from the Sigma-Aldrich (USA). Rhodaminephosphatidylethanol amine (Rh-PE), used for the modification of liposome membrane, was purchased from Molecular Probes (MO, USA). Water soluble carbodiimide hydrochloride (WSC, 1-ethyl-3-(3-dimethylaminopropyl) carbodiimide hydrochloride), calcein, and HOBt (1-Hydroxy-1H-benzotriazole, monohydrate) were purchased from Dojindo Chemicals (Tokyo, Japan). Other reagents of analytical grade were purchased from Wako.

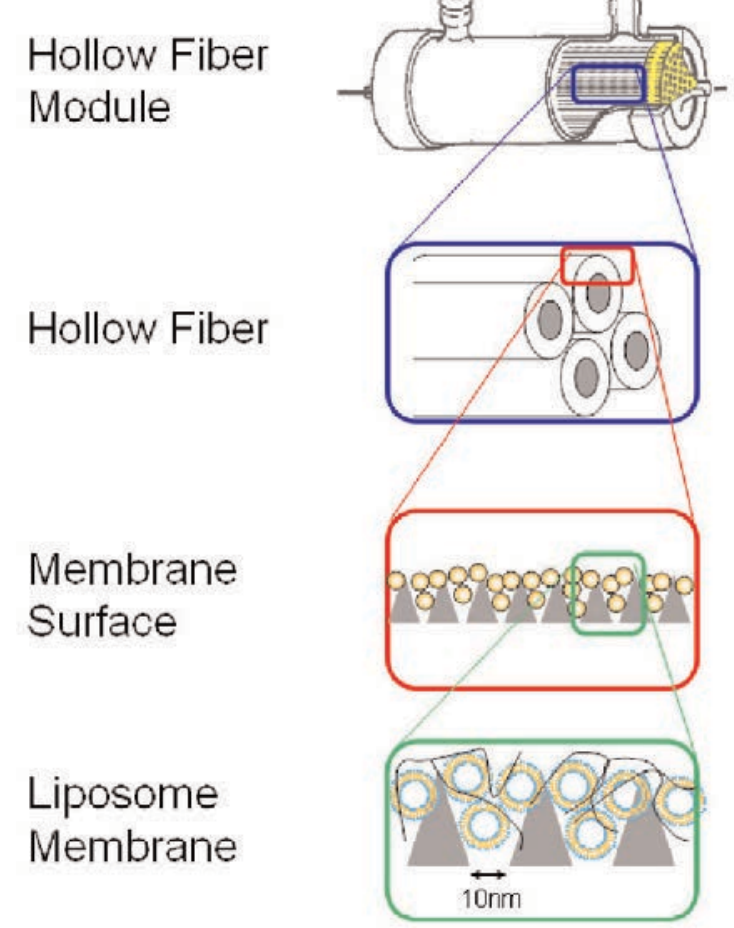

Fig. 1 Conceptual illustration of immobilized liposome membrane module with follow fibers.

\subsection{Liposome Preparation}

The POPC liposome including $1 \mathrm{~mol} \% \mathrm{Rh}-\mathrm{PE}$ and 1 mol\% biotinyl-phosphatidyl ethanolamine was prepared by using the following procedure according to the previous work ${ }^{5}$. For the preliminary experiment to test the clearance of the module, the POPC liposome including $30 \mathrm{~mol} \%$ cholesterol was also prepared. The phospholipid was dissolved in chloroform/methanol. After the solvent was evaporated, the resulting thin film was dried for at least $2 \mathrm{~h}$ under a vacuum. The lipid film was hydrated by pure water or $100 \mathrm{mM}$ calcein solution to form the multilamellar vesicles. The solution of the multilamellar vesicle was frozen in dry ice-ethanol $\left(-80^{\circ} \mathrm{C}\right)$ and incubated in the water bath above the phase-transition temperature. The above freezing-thawing treatment was repeated five times to prepare multi-lamellar vesicles (MLVs) with larger size. In order to prepare the single unilamellar vesicle with constant size, the MLVs were ultrasonicated to adjust the liposome size as $30 \mathrm{~nm}$ (for the preliminary experiment to confirm the clearance property of the module against the liposome solution) or were treated with the extrusion device equipped with stacked polycarbonate filters of $100-\mathrm{nm}$ pore size (for the immobilization experiment in membrane module). 


\subsection{Gel Preparation}

The hydrogel of anionic polymer and cationic PEI was prepared by using the amino coupling method. After the anionic polymer solution (0.25 wt $\%$ ), such as xanthan gum (XG), polyacyrylate (PA), and hiarilic acid (HA), was activated with WSC $(11 \mathrm{mg} / \mathrm{ml})$ and $11 \mathrm{mg} / \mathrm{ml} \mathrm{HOBt}$, the PEI solution at different concentrations $(0.1 \sim 1 \mathrm{wt} \%)$ was mixed with the above anionic polymer solution at room temperature. The water contents of obtained hydrogel were analyzed by calculating the weight of the hydrogel before and after the drying. In the case of the hydrogel formation on the membrane of the hollow fiber module, the POPC liposome was first loaded into the hollow fiber membrane. After the clearance of the liposome solution was confirmed, anionic polymer (XG (0.5 wt\%)) activated by $\mathrm{WSC} / \mathrm{HOBt}$ was then loaded into the membrane module for 20 min and, secondly, the PEI solution was applied to the membrane module for $30 \mathrm{~min}$.

\subsection{Membrane Module Operation}

Hollow fibers were obtained from a commercial polysulfone (PS) dialyzer (Toraysulfone TS-U, Toray, Japan). This commercial dialyzer was broken up to retrieve the hollow fibers. A mini module, used for the housing of the immobilized liposome membrane, was prepared by using the retrieved hollow fibers (100 fiber membranes). The membrane module was connected to the silicone tube with the inner diameter of $1 \mathrm{~mm}$ with the length of $130 \mathrm{~cm}$. The total volume of the module and the lines were determined by the preliminary experiment and was found to $3 \mathrm{ml}$. The peristaltic pump was equipped in the flow line of silicone tube. A manometer was set at the side of the filtrate. Before the sample loading, the water solution was applied to avoid the possible contamination by the impurities.

\subsection{Measurements}

The concentration of the liposome was monitored from the optical density of the solution at $405 \mathrm{~nm}$ $\left(\mathrm{OD}_{405}\right)$. The amounts of leaked calcein loaded in the liposome were determined from the absorbance at 415 $\mathrm{nm}$. The total amount of calcein in the liposome immobilized in membrane module was determined from the calcein leakage after washing module by $1 \mathrm{wt} \%$ Triton $\mathrm{X}-100$ solution to disrupt the liposome structure.

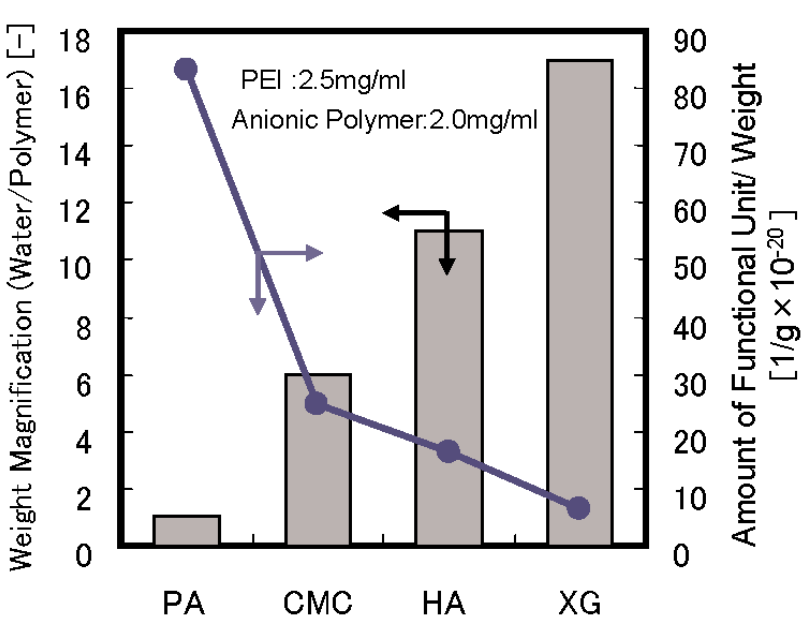

Fig. 2 Comparison of water contents of gel prepared with various polymers and PEI.

\section{Results and Discussion}

\subsection{Polyions-Conjugating Polymer Complex to Form Hydrogel}

The anionic polymers harboring $\mathrm{COOH}$ group can be conjugated with polycationic polymer harboring amine group via amino-coupling method. There are some anionic polymers which are known as a biocompatible polymers in respect to the hydrophilicity and also the high water contents in the formed gel. The preparation and characterization of the conjugatedpolyion complex were carried out as the preliminay experiment to prepare the immobilized liposome membrane module.

\subsubsection{Effect of Polymer Type on Hydrogel Preparation}

By using several kinds of polymers, such as polyacrylate (PA), carboxymethyl-cellulose (CMC), hyalurolic acid (HA), and also xanthan gum (XG), the hydrogel was formed by using polyethylene imine (PEI) as an amino-donating polymer through the amino coupling method using WSC. Fig. 2 shows the water contents of the gel prepared by using different kinds of anionic polymers and PEI at its same concentration. Among the possible polymers, the maximal values was obtained in the case of the $\mathrm{XG}$ and the value was reduced in the following order: $\mathrm{XG}>\mathrm{HA}>\mathrm{CMC}>$ PA. From the observation of the prepared of hydrogel, the above water contents could also be related to the stiffness of the hydrogel. For example, although the PAA/PEI gel showed solid-like nature, the HA/PEI 
(a)

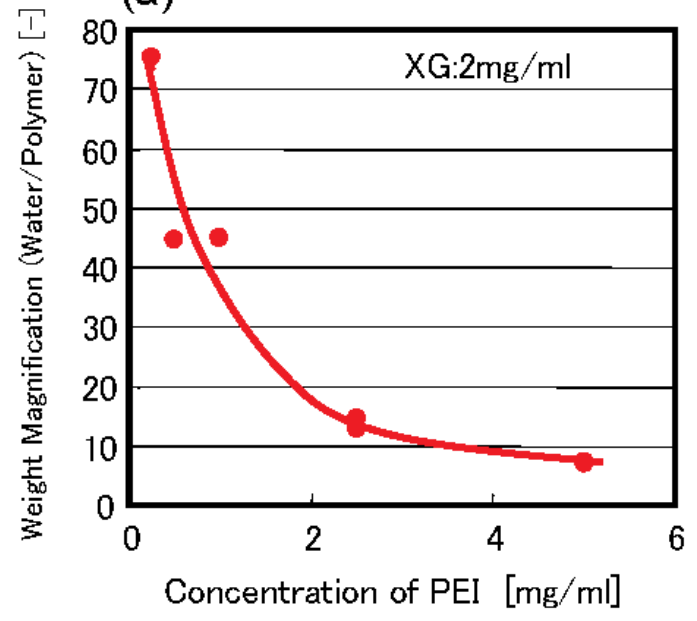

(b)

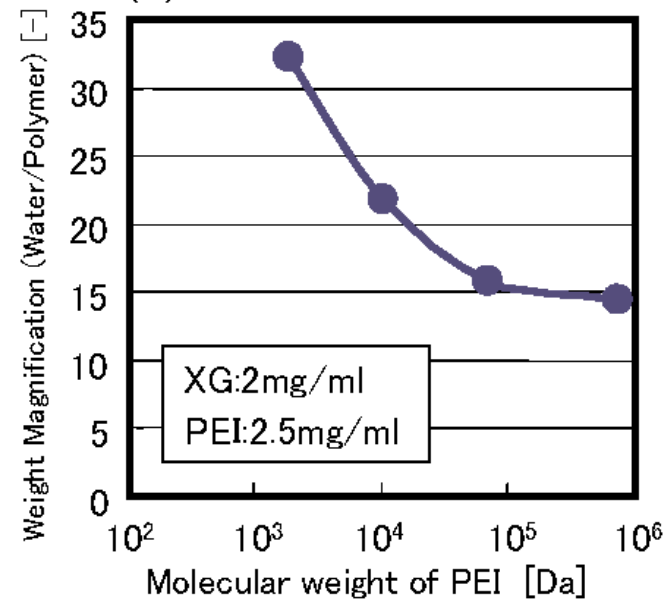

Fig. 3 Effect of operational condition on the water contents of XG/PEI Gel.

gel and XG/PEI gel were found to be soft. However, the HA/PEI gel became hard after the completion of the amino-coupling reaction for more than four hours.

Such tendency could be cuased by the density of the carboxyl-group on the surface of single polymer. PAA has a $\mathrm{COOH}$ group per single monomer unit, implying that the multi-conjugation points could be formed on the single polymer surface. The $\mathrm{COOH}$ density of $\mathrm{CM}$ Cellulose is also high because all the $\mathrm{OH}$-groups on $\mathrm{CMC}$ are substituted by carboxy methyl group. On the contrary, both HA and XG are known to be utilized as hydrophobic polymer and have a lower $\mathrm{COOH}$ density per monomer unit of the polymers: one and half $\mathrm{COOH}$ groups per two glucan moleculets (two glucosaminoglucan) in the case of $\mathrm{HA}$ and one $\mathrm{COOH}$ group per branched five glucan structure. The theoretical density of the $\mathrm{COOH}$ group in the single unit of the polymer was also plotted in Fig. 2. The $\mathrm{COOH}$ density was well corresponding with the water contents of the gel consisting of the polymers and the PEI.

It was thus found that the $\mathrm{XG} / \mathrm{PEI}$ gel was optimal as a soft-polymer interface to modify the PS membrane module. The XG/PEI gel was used in the following experiment.

\subsubsection{Effect of Operational Condition for XG/PEI Gel Preparation}

The effect of the preparation condition on the $\mathrm{XG} / \mathrm{PEI}$ gel formation was investigated. The obtained results were shown in Fig. 3. As shown in Fig. 3(a), the effect of the PEI concentration on the water contents of the hydrogel was first investigated. At the lower concentration region of PEI, the hydrogel was not formed at all. Above the critial PEI concentration $(0.25 \mathrm{mg} / \mathrm{ml})$, the XG/PEI gel was formed. It has been reported that the XG and PEI solution could form the polyion complex in the aquous solution because of the formation of hydrophobic and less soluble precipitation $^{31}$. This critial concentration to form the hydrogel is related with the phase forming behaviors of the polymer solution. The water contents of the hydrogel decreased with the increase of the PEI concentration. The effect of the molecular weight of the PEI on the water contents was further investigated at the constant concentration of PEI monomer unit. Fig. 3(b) shows the relationship between the molecular weight of PEI and the water contents of the formed hydrogel. The water content with lower molecular weight of PEI was found to be much higher than that with higher molecular weight. As discussed in the previous section, the hydrogel could show solid-like nature through the increase of the cross-linking points between the polymers. The above results on the effect of molecular weight of PEI imply that the concentration of the polymers as cross-linker could also be related to the stiffness of the formed hydrogel. The effect of the addition of liposome itself, to be immobilized on the hollow fiber module, on the water contents of the hydrogel was investigated. The addition of the liposome did not affect the water content of the XG/PEI gel (data not shown).

It was thus found that the $2 \mathrm{mg} / \mathrm{ml} \mathrm{XG}$ and 2.5 $\mathrm{mg} / \mathrm{ml}$ PEI could be effective for the appopreate gel formation in water solution considering the water contents of the prepared gel. 
(a) Chemical Structure of Polymer

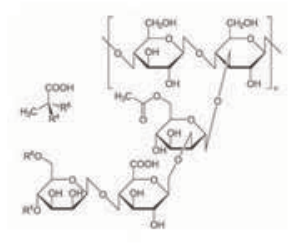

CarboxylTerminated Polymer (Xantan Gum)

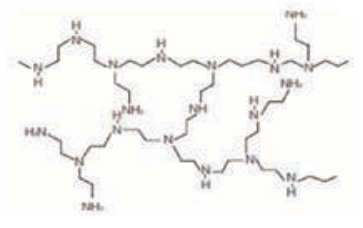

Amino-Terminated Polymer (Polyethylenelmine) (b) Module Setup

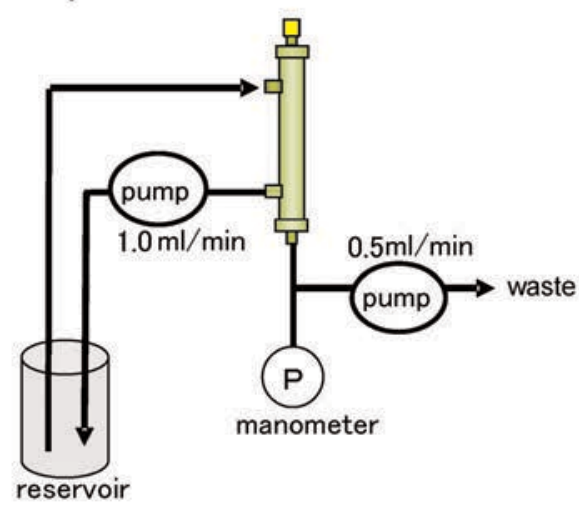

(c) Operational Scheme

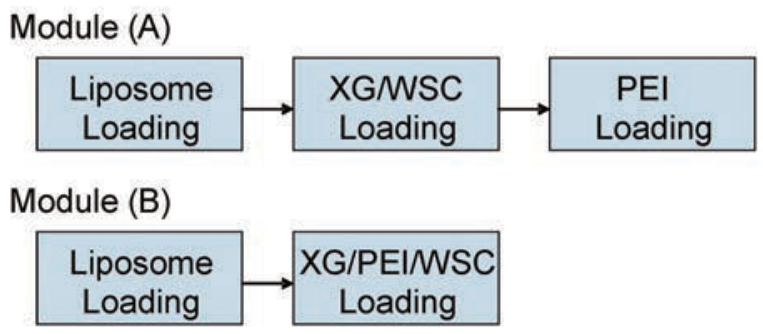

Fig. 4 Polymer cross-linking method for liposome immobilization using hollow fiber membrane with gradient size exclusive pore.

\subsection{Preparation of Immobilized Liposome Membrane Module}

The immobilized-liposome membrane module, schematically shown in Fig. 1, was prepared based on the above pre-investigation on the hydrogel formation. The operational scheme for the liposome immobilization was shown in Fig. 4. The dialysate side (outside of the fiber membrane) of the membrane module was connected to the reservoir including samples such as liposome and polymers, where this flow line was equipped with peristaltic pump and manometer (Fig. 4(a)). This is because the wide cavity of the channel existed in this dialysate side. The filtrate was recovered from the blood side (inside of the fiber membrane) through the flow line equipped with peristaltic pump (Fig. 4(a)).

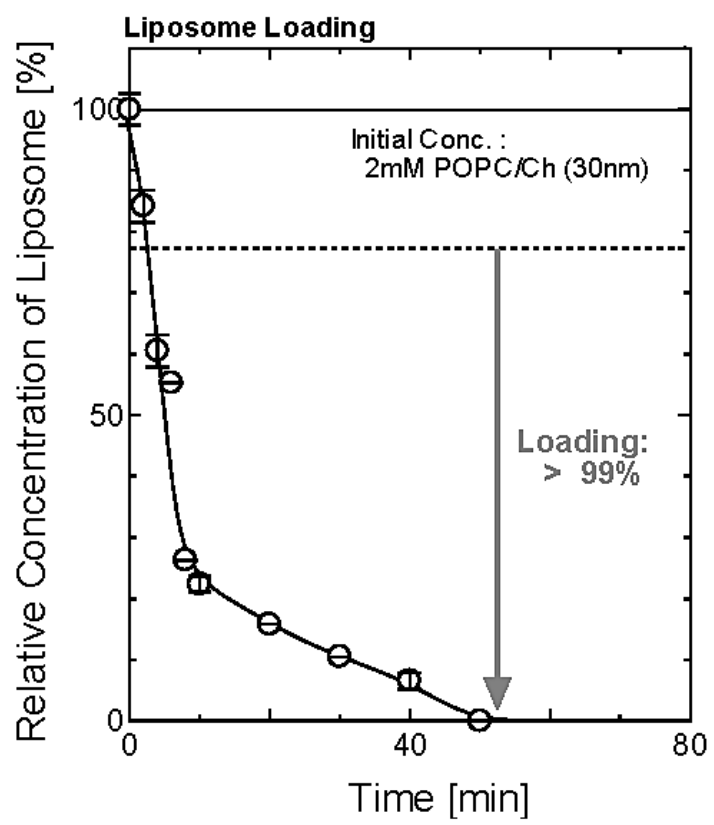

Fig. 5 Typical example of the clearance (entrapment) of liposome in hollow fiber membrane module as a function of loading time. The liposome clearance in the membrane module was studied as a preliminary experiment prior to the liposome immobilization experiment. Flow rate was set at $1.0 \mathrm{ml} / \mathrm{min}$. Total volume of the module and flow tubes was $3 \mathrm{ml}$ and the sample volume was $10 \mathrm{ml}$, resulting in the 0.78 dilution of sample at infinite time. Liposome used here was $2 \mathrm{mM}$ POPC/Ch $(30 \mathrm{~nm})$.

The POPC liposome with $100 \mathrm{~nm}$ was first loaded the PS membrane module from the dialysate side (from housing side) with circulation mode (Fig. 4(b) Step I), confirming that all the liposome (more than $99 \%$ was cleared in $40 \mathrm{~min}$ at $1 \mathrm{ml} / \mathrm{min}$ from the reserver based on the optical density (a typical example was shown in Fig. 5). The aqueous solution of XG $(1 \mathrm{mg})$ activated by WSC was then applied to the module for 20 min to fill the empty space between the liposomes on the PS membrane surface. Almost all XG molecules were entrapped on the PS membrane because of its large molecular weight (average moleular weight: $2000 \mathrm{kDa}$ ) (Fig. 4(b) Step II). At the final stage, the aqueous solution of PEI was loaded to the module in order to form the XG/PEI gel on the surface of PS membrane (Fig. 4(b) Step III). After this operation, the pressure drop between the PS membrane was increased from $2.0 \mathrm{kPa}$ to $4.7 \mathrm{kPa}$, showing that the gel was formed inside a channel of the PS membrane. Although the resistance of the water filtration was increased after the gel formation, the value was found 
to be high enough to be utilized for the reactor and/or separator.

The photo image of the immobilized liposome membrane module was shown in Fig. 6. The POPC liposome was herewith colored with fluorescence probes, such as $100 \mathrm{mM}$ calcein (entrapped inside inner aqueous phase, Orange Color) and Rhodamine-PE (modified with membrane itself at $2 \mathrm{~mol} \%$, Red Color) in order to estimate the immobilized amounts of liposome. In contrast to the normal membrane module (Fig. 6, Control), the fluorescence-labelled liposome was observed in the hollow fiber module (Fig. 6, Module (A)), clearly showing that the liposome was entrapped on the PS membrane surface. In addition, the back pressure was applied from the inner side of the membrane fiber to outer side for $20 \mathrm{~min}$. A significant change in the fluorescence was not observed although the liposome loaded without the formation of $\mathrm{XG} / \mathrm{PEI}$ gel was leaked from the membrane module under such a back pressure condition (data not shown). The result on the back pressure experiment shows that the liposome was entrapped inside the gel matrix of XG/PEI. As a negative control experiment, the gel formation was performed without sequential addition of XG (Step II) and PEI (Step III), where the $\mathrm{XG} / \mathrm{PEI}$ mixture was applied to the module after the liposome loading (Step I). Although the color of calcein and Rhodamine-PE was observed similarly, the fluorescence color was not deep (Fig. 6, Module-(B)).

\subsection{Characterization of Immobilized Liposome Membrane Module}

The obtained membrane module immobilizing the liposome membrane (HF-ILM) was further characterized, focusing on the (i) immobilized amount of the liposome, (ii) stability of the immobilized liposome and (iii) filtration constant of the membrane module itself.

The calcein was used as a measure of liposome immobilization because the calcein can be easily released by the addition of an excess amount detergent. The amount of calcein leakage is proportional to the concentration of liposome entrapped because the calcein concentration inside the liposome is constant at $100 \mathrm{mM}$. The Triton $\mathrm{X}-100$ solution ( $1 \mathrm{wt} \%$ ) was applied to the membrane module for the solubilization of the liposome membrane and the total amounts of calcein leakage were measured as shown in Table 1. The calcein leakage of sequential immobilization (Fig.

\section{Control}

\section{Module \\ (A)}

Module

(B)

Fig. 6 Immobilized liposome membrane module prepared with different conditions. $100 \mathrm{mM}$ calcein was entrapped in POPC Liposome $(100 \mathrm{~nm})$ modified with $1 \mathrm{~mol} \%$ Rhodamine-PE. The liposome solution ( $2 \mathrm{ml}, 30 \mathrm{mM}$ lipid) was applied to the membrane module. The XG/PEI gel was formed through the loading of activated polymers (A) stepwise and (B) simultaneously.

6, Module (A)) was found to be 10 times higher than that of the non-sequential immobilization (Fig. 6, Module (B)). This could be because of the disruption of the liposome during the XG/PEI gel formation. In the case of the sequential loading of the polymer, the XG polymer was first packed inside a channel and the PEI closss-links the packed XG gel, resulting that the liposome was not disrupted during this operation.

The result on the immobilized liposome, determined from the calcein leakage, was compared with previous data on the immobilized liposome chromatography (Table 1). The amount of immobilized lipid shows 820 $\mu \mathrm{mol} / \mathrm{ml}-\mathrm{PS}-$ membrane and is $27 \sim 40$ times larger than that in the case of immobilized liposome hydrogel ${ }^{32,33)}$. The difference of the present membrane module and previous immobilized liposome gel matrix is caused by the difference of the immobilization method. In the case of the immobilization of liposome on the gel matrix, the surface area of the gel surface to be immobilized was restricted ${ }^{32}$. However, the present method enables us to immobilize the liposome because the liposome was first loaded into hollow fiber module with highly porous membrane. In addition, various kinds of liposome could also be immobilized by using this method because the loaded liposome was physically immobilized through the creation of gel matrix in between the liposomes.

The stability of the liposome immobilized on the PS membrane surface is also important factor for the practical use ${ }^{32,33}$. The time course of the calcein leakage was monitored after the above HF-ILMs were pre- 
Table 1 Characteristics of Liposome-Immobilized Matrix

\begin{tabular}{|c|c|c|c|c|c|}
\hline & Matrix Type & Immobilization Method & $\begin{array}{c}\text { Amount of } \\
\text { Immobilized Lipid } \\
{[\mu \mathrm{mol}-\mathrm{lipid} / \mathrm{ml}-\text { matrix }]}\end{array}$ & $\begin{array}{c}\text { Stability } \\
\text { (Calcein Leakage ) } \\
{[\%]}\end{array}$ & $\begin{array}{c}\text { UFRS } \\
{\left[\mathrm{ml} /\left(\mathrm{hr} \mathrm{m}^{2} \mathrm{kPa}\right)\right]}\end{array}$ \\
\hline Control Module & PS Membrane & None & 0 & - & 850 \\
\hline ILM -Module -(A) & PS Membrane & XG/PEI(Sequential) & $820.0(* 1)$ & $0.89(* 5)$ & 230 \\
\hline ILM -Module -(B) & PS Membrane & $\mathrm{XG} / \mathrm{PEI}$ (Preforming $\quad-\mathrm{Gel})$ & $49.1(* 1)$ & $72.0(* 5)$ & 410 \\
\hline ILC & Sepharose & Avidin-Biotin & $37.6(* 2)$ & $0.53(* 6)$ & - \\
\hline ILC & Sephacryl & Avidin -Biotin & $41.5(* 2)$ & $0.77(* 6)$ & - \\
\hline ILC & Sephadex & Avidin-Biotin & $30.2(* 2)$ & $1.08(* 6)$ & - \\
\hline ILC & TSK & Avidin-Biotin & $30.3(* 2)$ & $0.74(* 6)$ & - \\
\hline ILC & TSK & Covalent Binding & $34.2(* 3)$ & - & - \\
\hline ILM & Cellulose & Covalent Binding & $14.5(* 4)$ & $81.2(* 7)$ & - \\
\hline
\end{tabular}

*1 The amount of the immobilized lipid was calculated from the calcein leakage after the wash of the non-entrapped calcein outside the liposome by buffer solution and the solubilization of immobilized liposome by using Triton X-100 solution. Triton $\mathrm{X}-100$ solution more than its critical micelle concentration is known to disrupt the liposome structure, resulting in the leakage of the calcein from the immobilized liposomes. The total amount of calcein leaked from the module is equivalent to the total calcein entrapped inside the liposome immobilized in the module. The number of liposomes can be estimated from the total amounts of leaked calcein because the calcein concentration inside a liposome is set at $100 \mathrm{mM}$. The number of lipid molecules of one liposome (size: $100 \mathrm{~nm}$ ) can be calculated as 79725 by assuming $0.72 \mathrm{~nm}^{2}$ as surface area of single lipid and $4.5 \mathrm{~nm}$ as the bilayer thickness. The amount of the lipid can be calculated based on the number of liposome, immobilized in the membrane module ( total amount of leaked calcein) and the number of the lipid molecules of one liposome. For example, the amount of lipid, loaded into the module, was calculated as $57.4 \mu$ mol in the case of ILM-Module-(A). The value was confirmed to be almost equivalent to that calculated from the initial lipid amount (60 $\mu$ mol) of feed solution of liposome (30 mM lipid, $2 \mathrm{ml}$ ). The amount of the immobilized lipid in the membrane matrix was finally calculated based on the total volume of polymer material of hollow fiber membranes $(0.07 \mathrm{ml})$.

*2 Yang Q, Liu X-Y, Ajiki S-I, Hara M, Lundahl P, Miyake J : J. Chromatogr. B, 707, 131-141 (1998)

*3 Yang Q, Liu X-Y, Yoshimoto M, Kuboi R, Miyake J : Anal. Biochem., 268, 354-362 (1999)

*4 Data from Unpublished Work

* 5 The value was calculated as the accumulated amounts of calcein for several days at $25^{\circ} \mathrm{C}$ against its total amounts

*6 The value was recalculated based on the previously-reported data (Yang Q, Liu X-Y, Ajiki S-I, Hara M, Lundahl P, Miyake J : J. Chromatogr. B, 707, 131-141 (1998))

$* 7$ The value was recalculated based on data from unpublished work

pared as shown in Fig. 7. The calcein leakage was not observed in the case of module although the calsein was leaked in the case of module B. The calcein leakage from the immobilized liposome in gel matrix has previously been reported, where the calcein leakage was not observed at least for 3 weeks ${ }^{32}$. In the case of the liposome immobilized in the extremely porous membrane (cellulose acetate membrane), the life time of the calcein leakage was not so high (5 days) owing to the high shear stress of the liquid flow across the filter membrane (data not shown). The immobilization of the liposome on the SAM-supported solid surface was also performed and the immobilized liposome was found to be ruptured in one week. The lifetime of the present HF-ILM was found to be at least two weeks without significant leakage of the calcein. The above results show that the stability of the immobilized liposome is applicable for the analytical use and, also, preparation use.

The filtration constant was also shown in Table 1. The value was slightly reduced in the case of immobilized liposome PS module. It has been reported that $230 \mathrm{ml} /\left(\mathrm{hr} \mathrm{m}^{2} \mathrm{kPa}\right)$ in UFRS value is applicable for the dialysis use. The obtained value was lower than the 


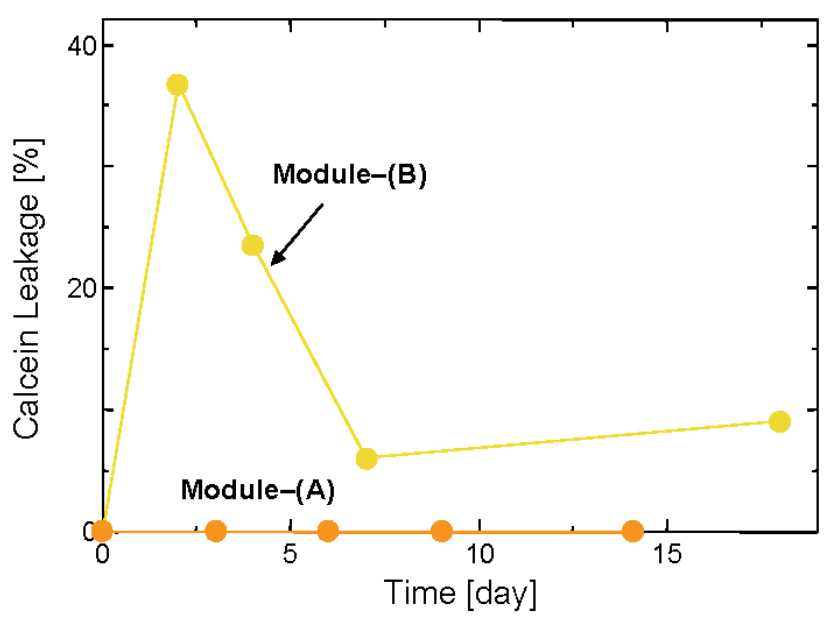

Fig. 7 Time course of leakage of calcein from the liposome immobilized on hollow fiber PS-membrane module.

maximal level of the filtration resistance, showing that this immobilized liposome module can be applied for the practical usage.

It was thus found that the prepared HF-ILM can be used in relation to the (i) immobilized amounts of liposome, (ii) its stability, and (iii) filtration nature.

\section{Conclusion}

The immobilized liposome membrane module was newly developed by employing the sequential immobilization technique of liposome and polymers for gel matrix. The XG/PEI gel was employed as a gel for the liposome immobilization. The immobilized liposome membrane module, prepared at optimal condition, was found to exceed in the following aspects: (i) high immobilization, (ii) high stability, (iii) high efficiency. Considering the immobilization principle, this method could be applied for the immobilization of various kinds of liposome. It has previously reported that the liposome has a molecular chaperone-like function ${ }^{5 \sim 7)}$ and the immobilized liposome gel column was applied to the protein refolding column ${ }^{1)}$. Recently, the liposome could induce the enzyme-like function (LIPOzyme) ${ }^{12 \sim 19)}$. The previous findings show that the liposome could be utilized as a catalytic material. The combination of the liposome and/or LIPOzyme with the present immobilized liposome membrane module could enable us to develop the liposome-based bioreactor. In addition, the liposome immobilization could change the biocompatibility of the membrane surface itself. The obtained membrane module could contribute to the possible applications shown above. The possible applications are now under investigation.

\section{Acknowledgement}

The fundamental concept of this study was supported by the Research Group of "Membrane Stress Biotechnology" and "Engineering Science of Liposome". It is partly supported by a Grant-in-Aid for Scientific Research (No. 15206089, 16686046, 16760635, 17656268, 19656203, 19656220, and 20360350, 20760539, 21246121) from the Ministry of Education, Science, Sports, and Culture of Japan, a grant from the 21st Century COE program "Creation of Integrated EcoChemistry" and the Global COE program "Bio-Environmental Chemistry" of the Japan Society for the Promotion of Science (JSPS). The authors are grateful to the Research Center for Solar Energy Chemistry of Osaka University and the Gas hydrate Analyzing System of Osaka University.

\section{Literature Cited}

1) Kuboi R, Yoshimoto M, Shimanouchi T, Umakoshi H : J. Chromatogr, B, 743, 93-99 (2000)

2) Yamahara K, Ota H, Kuboi R : J. Chem. Eng. Japan, 31, 795-803 (1998)

3) Kuboi R, Umakoshi H : Solv. Extr. Res. Dev. Japan, 13, 921 (2006)

4) Kuboi R, Umakoshi H, Shimanouchi T : Membrane, 33, 266-306 (2008)

5) Kuboi R, Yoshimoto M, Walde P, Luisi PL : Biotechnol. Progr., 13, 826-836 (1997)

6) Yoshimoto M, Kuboi R : Biotechnol. Progr., 15, 480-487 (1999)

7) Umakoshi H, Yoshimoto N, Yoshimoto M, Shimanouchi T, Kuboi R: Membrane, 32, 287-293 (2007)

8) Umakoshi H, Yoshimoto M, Shimanouchi T, Kuboi R, Komasawa I : Biotechnol. Progr., 14, 218-226 (1998)

9) Yoshimoto M, Walde P, Umakoshi H, Kuboi R : Biotechnol. Progr., 15, 689-696 (1999)

10) Menayame MF, Umakoshi H, Shimanouchi $T$, Yoshimoto M, Kuboi R : Biochem. Eng. J., 12, 7-19 (2002)

11) Menayame MF, Umakoshi H, Shimanouchi $T$, Yoshimoto M, Kuboi R : J. Biosci. Bioeng., 93, 498-501 (2002)

12) Nagami H, Umakoshi H, Shimanouchi T, Kuboi R : Biochem. Eng. J., 21, 221-227 (2004)

13) Nagami H, Yoshimoto N, Umakoshi H, Shimanouchi T, Kuboi R: J. Biosci. Bioeng., 99, 423-428 (2005)

14) Yoshimoto N, Tasaki M, Shimanouchi T, Umakoshi $H$, Kuboi R: J. Biosci. Bioeng., 100, 455-459 (2005)

15) Tuan LQ, Umakoshi H, Shimanouchi T, Kuboi R : 


\section{Langmuir, 24, 350-354 (2008)}

16) Umakoshi H, Morimoto K, Ohama Y, Nagami H, Shimanouchi T, Kuboi R : Langmuir, 24, 4451-4455 (2008)

17) Tuan LQ, Umakoshi H, Shimanouchi T, Kuboi R : Enzym. Microb. Technol., 44, 101-106 (2008)

18) Tuan LQ, Umakoshi H, Shimanouchi T, Kuboi R : Memebrane, 33, 173-179 (2008)

19) Bui HT, Umakoshi H, Ngo KX, Nishida M, Shimanouchi T, Kuboi R : Langmuir, 24, 10537-10542 (2008)

20) Shimanouchi $T$, Morita S, Umakoshi H, Kuboi R : J. Chromatogr. B, 743, 85-91 (2000)

21) Jung HS, Ishii $H$, Shimanouchi $T$, Umakoshi H, Kuboi R : Membrane, 32, 294-301 (2007)

22) Jung HS, Umakoshi H, Son S-Y, Shimanouchi T, Kuboi R: Solv. Extr. Res. Dev. Japan, 10, 123-132 (2003)

23) Morita S, Umakoshi H, Kuboi R : J. Biosci. Bioeng., 90, 157-162 (2000)

24) Yang Q, Lundahl P : Anal. Biochem., 218, 210-221 (1994)

25) Yang Q, Liu X-Y, Ajiki S-I, Hara M, Lundahl P, Miyake $\mathrm{J}:$ J. Chromatogr. B, 707, 131-141 (1998)

26) Hara M, Yuan H, Miyake M, Iijima S, Yang Q, Miyake J : Mat. Sci. Eng. C, 13, 117-121 (2000)

27) Yang Q, Liu X-Y, Yoshimoto M, Kuboi R, Miyake J : Anal. Biochem., 268, 354-362 (1999)

28) Gabelman A, Hwang S-T : J. Membrane Science, 159, 61106 (1999)

29) Yang Q, Liu X-Y, Yoshimoto M, Kuboi R, Miyake J : Anal. Biochem., 268, 354-362 (1999)

30) Tanaka Y, Akiyama H, Kuroda T, Jung G, Tanahashi K, Sugaya H, Utsumi J, Kawasaki H, Hirano H : Proteomics, 6 (17), 4845-4855 (2006)

31) Hsu CH, Chu YF, Argin-Soysal S, Hahm TS, Lo YM : J. Food Science, 69, E441-E448 (2004)

32) Liu X-Y, Nakamura C, Nakamura N, Hirano T, Shinbo T, Miyake J : J. Chromatogr. A, 1087, 229-235 (2005)

33) Liu X-Y, Yang Q, Nakamura C, Miyake J : J. Chromatogr. $B$, 750, 51-60 (2001)

(Received 26 December 2008; 